\title{
Nuevas perspectivas para la intervención en barriadas protegidas. En búsqueda de comunidades resilientes
}

\author{
Daniel Navas-Carrillo | Dpto. Urbanística y Ordenación del Territorio, Universidad de Sevilla
}

URL de la contribución <www.iaph.es/revistaph/index.php/revistaph/article/view/3818>

Numerosas barriadas de vivienda social que se han construido en Andalucía en la segunda mitad del siglo XX han sido incluidas en los catálogos de protección del planeamiento de sus respectivas ciudades. Construidas ante las "urgentes necesidades de viviendas en Europa al finalizar las guerras de mitad de siglo como respuesta al trasvase de población campo-ciudad" (MOLINA COSTA; RUBIO DEL VAL, 2010: 16) son "referentes históricos de una política de vivienda concreta del pasado" (PÉREZ CANO; MOSQUERA ADELL, 2006: 115). Muchas de ellas han sido incluidas además en el Registro Andaluz de Arquitectura Contemporánea (RAAC) como ejemplos significativos de la Arquitectura del Movimiento Moderno en la comunidad.

Es indudable que el reconocimiento, difusión, y especialmente su protección ha permitido mantener en los años de mayor presión inmobiliaria muchos de los valores patrimoniales por los que fueron declarados. Sin embargo, presentan altos índices de obsolescencia en su respuesta a los estándares de confort exigidos por la ciudadanía actual. Si bien es cierto que la administración, y especialmente la andaluza, ha jugado en los últimos años un papel decisivo en su recuperación mediante la financiación -casi en exclusiva- de las operaciones de regeneración de estas barriadas (SALMERÓN ESCOBAR; RODRÍGUEZ GALADÍ, 2010).

Esta trayectoria ha puesto sobre la mesa la necesidad de aportar nuevas perspectivas que superen el hecho puntual de la rehabilitación. Como principal objetivo, se debe evitar que estos conjuntos urbanos, una vez adaptados a las exigencias actuales, sufran nuevos procesos de obsolescencia en el futuro. Esta meta lleva implícito el aumento de la capacidad -como colectivo- para resistir y adaptarse de forma continuada a aquellas condiciones adversas sobrevenidas en un contexto como el actual en continua transformación, dotando al usuario de un papel activo en la conservación de estos bienes. El reto pasa por trascender las políticas actuales en materia de regeneración urbana desde prácticas que tengan como objeto reforzar el sentimiento de comunidad y el empoderamiento de la ciudadanía. Se trata de uno de los principales cambios de paradigma que se están produciendo en la contemporaneidad en consonancia con el objetivo de la optimización del tiempo, del espacio y de los recursos materiales y económicos a emplear que subyacen de la Estrategia Europa 2020 para un crecimiento inteligente, sostenible e integrador (COMISIÓN EUROPA, 2010).

Esto no quiere decir, que las intervenciones que se han venido realizando no hayan llevado aparejado procesos participativos en paralelo. Lo que si es cierto que estos han sido utilizados -casi exclusivamente- como metodologías aproximativas de análisis y diagnóstico de las necesidades. En este sentido, la nueva visión propuesta aboga por atender a cuestiones referidas a la construcción social de nuestro hábitat, bien desde la dimensión cultural en cuanto a lo identitario, como desde las estructuras organizativas que se establecen como propias de cualquier comunidad. Para ello se propone la traslación del concepto de resiliencia sobre la condición social de lo patrimonial.

La definición y aplicación de este término, originalmente analizado desde el ámbito de la ecología por los trabajos de HOLLING (1973), ha caminado en paralelo a la evolución y profundización sobre las múltiples dimensiones de la sostenibilidad, tal como señala MALLQUI SHICSHE (2012). Se transciende así el ámbito exclusivamente de lo ambiental, para aplicar también el concepto sobre las estructuras sociales, en autores como FOLKE (1996). Desde esta visión, la resiliencia puede definirse como 
a debate Relaciones entre ciudadanía y agentes patrimoniales. Colaboración, conflicto y mediación

| coordinan Cristina Sánchez-Carretero, Guadalupe Jiménez-Esquinas

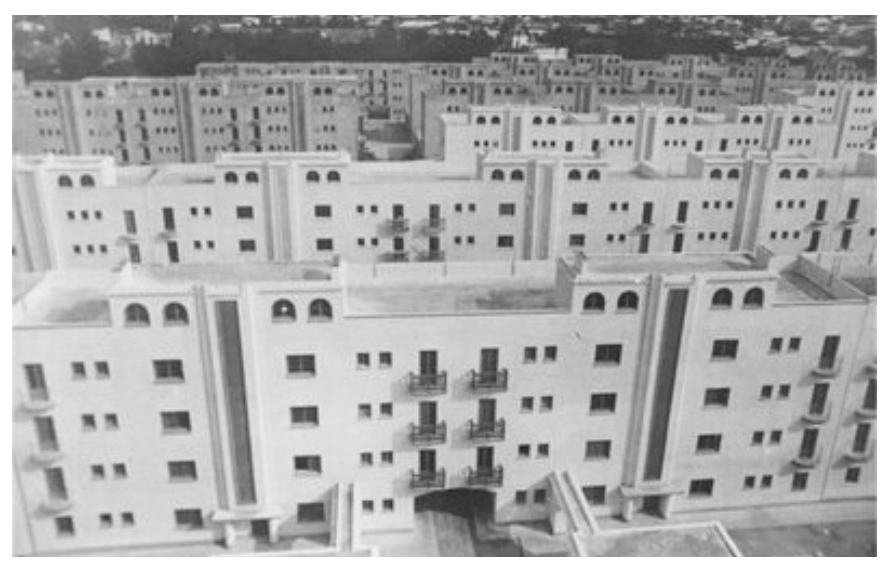

Proyecto de la barriada de la Plata en Jerez de la Frontera (Cádiz) | fuente RAAC

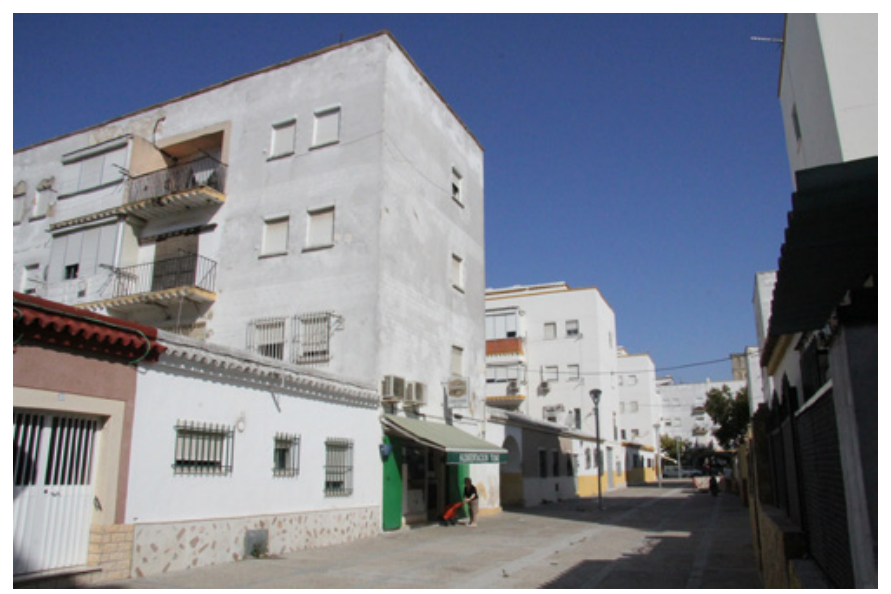

Estado actual de la barriada de la Plata en Jerez de la Frontera (Cádiz). 2011 | fotos Fondo Gráfico IAPH (Plácido González Martínez)

"la capacidad de las comunidades para resistir shocks externos a su infraestructura social" (ADGER, 2000, 347) al tiempo que es la "capacidad que tiene un sistema, formado a partir del individuo hasta la totalidad de la comunidad de mantener la unión y conservar la capacidad de funcionamiento" (BARR; DEVINE-WRIGHT, 2012). Por lo tanto, una comunidad resiliente puede ser definida como "aquella que asume una acción intencional para aumentar su capacidad personal y colectiva" (COLUSSI, 2000: 9).

La resiliencia aplicada sobre la condición urbana puede enfocarse desde dos visiones según lo establecido por
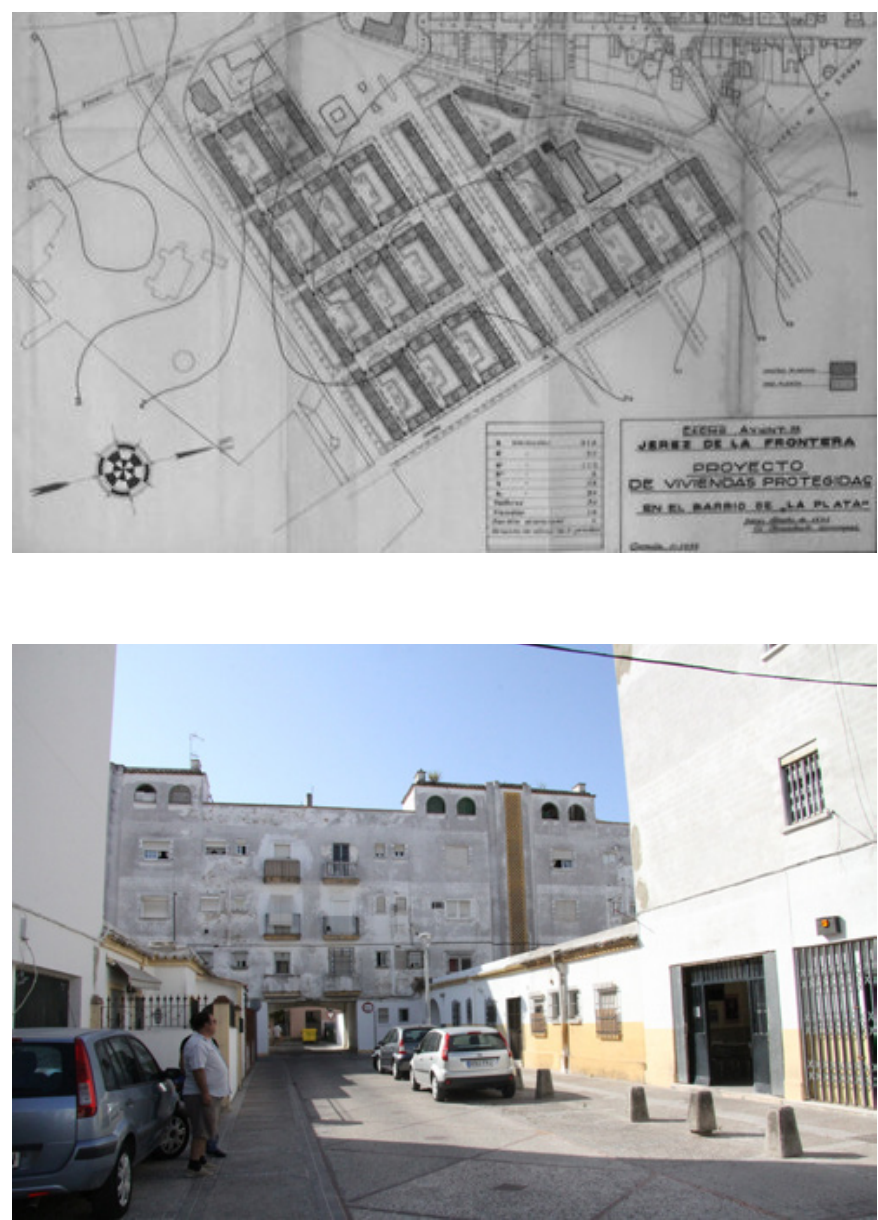

POLÉSE (2010). La primera tiene que ver con la capacidad que tiene una ciudad de reconstruirse después de alguna catástrofe, ya sea natural o provocada por el ser humano. Se trata de la visión más extendida en la literatura, pero que carece de aplicación en el ámbito abordado. Sin embargo, la segunda perspectiva, mucho menos estudiada, tiene relación directa con la capacidad intrínseca que tiene una comunidad para regenerar su tejido social y renovar sus espacios deteriorados. En este sentido, la resiliencia es la "la capacidad para resistir a crisis sistémicas de origen externo, pero reforzadas por ciertas debilidades locales, causantes de un proceso de declive a largo plazo que pone en cuestión su fun- 
cionalidad anterior, consiguiendo una transformación interna que permite su adaptación positiva al contexto imperante en la nueva etapa" (MÉNDEZ GUTIÉRREZ DEL VALLE, 2012: 219).

Estas definiciones están en consonancia con la evolución que ha sufrido el concepto de patrimonio en los últimos años, el cual ha transciendo las consideraciones arquitectónicas que tradicionalmente lo han definido, para incluir aspectos inmateriales que aboguen por el reflejo de la identidad como sociedad y el atractivo del bien como garantía de su supervivencia. Tal como se recoge en la Resolución de Arequipa ${ }^{1}$ más allá de las preocupaciones por los riesgos naturales, la defensa del patrimonio se justifica como recurso necesario en la definición del concepto de resiliencia en su aplicación al ámbito urbano desde la perspectiva económica y social. Por tanto, se puede concluir que el fomento de la capacidad resiliente de las comunidades que habitan el patrimonio residencial se vislumbra como prioridad para ser abordada en el diseño de nuevas políticas de gestión patrimonial.

\section{NOTA}

1. Resolución adoptada en el XIII Congreso Mundial de la Organización de Ciudades Patrimonio Mundial sobre Ciudades Resilientes, celebrado en la ciudad de Arequipa (Perú) del 3 al 6 de noviembre de 2015.

\section{BIBLIOGRAFÍA}

- ADGER, W. N. (2000) Social and Ecological Resilience: Are They Related? Progress in Human Geography, septiembre 2000, vol. 24, n. ${ }^{\circ} 3$, pp. 347-364

- BARR, S.; DEVINE-WRIGHT, P. (2012). Resilient communities: sustainabilities in transition. Local Environment: The International Journal of Justice and Sustainability, abril 2012, vol. 17, n. ${ }^{\circ}$, pp. 525-532
- COMISIÓN EUROPEA (2010) EUROPA 2020: Una Estra tegia para un crecimiento inteligente, sostenible e integrador. En EUR-Lex: Access to European Union law <http://eur-lex. europa.eu/legal-content/ES/TXT/?uri=URISERV\%3Aem0028> [Consulta: 26/07/2016]

- COLUSSI, M. M. (2000) The community resilience manual. A resource for rural recovery and renewal. Port Alvemit: Centre for Commnunity Enterprise, 2000

- FOLKE, C. (2006) Resilience: the emergence of a perspective for socio-ecological systems analyses. Global Environmental Change, agosto 2006, vol. 16, n. ${ }^{\circ} 3$, pp. 253267

- HOLLING, C. S. (1973) Resilience and stability of ecological systems. Annual Review of Ecology and Systematics, noviembre 1973, vol. 4, pp. 1-23

- MALLQUI SHICSHE, A. A. (2012) La planificación de la Gestión del Riesgo de Desastres. ¿Cómo desarrollar ciudades resilientes? Fractal Revista de Arquitectura de la Universidad Peruana Los Andes UPLA, mayo 2012, n. ${ }^{\circ} 1$, pp. 22-23

- MÉNDEZ GUTIÉRREZ DEL VALLE, R. (2012) Ciudades y metáforas: sobre el concepto de resiliencia urbana. Ciudad y Territorio. Estudios Territoriales, verano 2012, vol. XLIV, n. ${ }^{\circ}$ 172, pp. 215-232

- MOLINA COSTA, M.; RUBIO DEL VAL, J. (2010) Estrategias, retos y oportunidades en la rehabilitación de los polígonos de viviendas construidos en España entre 1940 y 1980. Ciudades Revista del Instituto de Urbanística de la Universidad de Valladolid, julio de 2010, n. ${ }^{\circ} 13$, pp. 15-37

- PÉREZ CANO, M. T.; MOSQUERA ADELL, E. (2006) La Protección del Patrimonio Edificado. Catalógo de Bienes Inmuebles del Municipio de Almonte. Sevilla: Secretariado de Publicaciones de la Universidad de Sevilla, 2006

- PÉREZ ESCOLANO, V.; FERNÁNDEZ-BACA CASARES, R. (coord.) (2012) Cien años de arquitectura en Andalucía: el Registro Andaluz de Arquitectura Contemporánea, 1900-2000. Sevilla: Instituto Andaluz del Patrimonio Histórico. Junta de Andalucía, 2012

- PLODINEC, M. J. (2009) Definitions of community resilience: an analysis. Washington: Community \& Regional Resilience Institute, 2009

- SALMERÓN ESCOBAR, F.; RODRíGUEZ GALADÍ, J. I. (2010) Rehabilitación integral de los barrios andaluces. En Ponencias del Congreso SB10mad. Edificación sostenible, Revitalización y Rehabilitación de barrios [en línea] <http://www. sb10mad.com/ponencias/archivos/a/A013.pdf> [Consulta: 20/ 7/2016] 